\title{
Using Functional Genetics to Understand Breast Cancer Biology
}

\author{
Alan Ashworth ${ }^{1}$ and Rene Bernards ${ }^{2}$ \\ ${ }^{1}$ The Breakthrough Breast Cancer Research Centre, The Institute of Cancer Research, Fulham Road, \\ London, SW3 6JB, United Kingdom \\ ${ }^{2}$ The Netherlands Cancer Institute, Plesmanlaan 121, 1066 CX Amsterdam, The Netherlands \\ Correspondence: alan.ashworth@icr.ac.uk and r.bernards@nki.nl
}

Genetic screens were for long the prerogative of those that studied model organisms. The discovery in 2001 that gene silencing through RNA interference (RNAi) can also be brought about in mammalian cells paved the way for large scale loss-of-function genetic screens in higher organisms. In this article, we describe how functional genetic studies can help us understand the biology of breast cancer, how it can be used to identify novel targets for breast cancer therapy, and how it can help in the identification of those patients that are most likely to respond to a given therapy.

M uch remains to be learned regarding the function of mammalian genes. Only some quarter of all human genes have well-described functions. It is likely that quite a few of these currently unannotated genes will turn out to play key parts in cancer biology. For example, a 70-gene gene signature that can discriminate breast tumors of good and poor prognosis contained some 20 genes of currently unknown function (van 't Veer et al. 2002). The fact that these genes of unknown function foretell breast cancer prognosis hints at a role for at least some of these genes in breast cancer biology. The unbiased search for genes that contribute to breast cancer development is therefore likely to yield a rich harvest of new insights. RNA interference allows us to suppress genes systematically on a large scale and study the effects of gene suppression on specific cellular processes or signaling pathways. Consequently, RNA interference-based genetic screens have the potential to deepen our understanding of the molecular events that cause breast cancer, to find novel targets for therapy and to find biomarkers of drug responsiveness. In this article, we will describe the technologies available to perform both gain-of-function and loss-offunction genetic screens and will illustrate how such functional genetic screens have been used in the recent past to study a variety of outstanding questions in the biology of breast cancer.

\section{LOSS-OF-FUNCTION GENETIC SCREENS}

The use of specific siRNAs and shRNAs generated against individual genes has been very important in allowing the functional dissection of essentially any cellular protein. The

Editors: Mina J. Bissell, Kornelia Polyak, and Jeffrey Rosen

Additional Perspectives on The Mammary Gland as an Experimental Model available at www.cshperspectives.org

Copyright (C) 2010 Cold Spring Harbor Laboratory Press; all rights reserved; doi: 10.1101/cshperspect.a003327

Cite this article as Cold Spring Harb Perspect Biol 2010;2:a003327 
A. Ashworth and R. Bernards

development of siRNA and shRNA libraries, allowing the parallel targeting of a wide range of genes, has made it possible to conduct highthroughput screens that systematically interrogate phenotypes associated with the silencing of gene expression on a large, up to genomewide, scale (Bernards et al. 2006; Iorns et al. 2007). Libraries of siRNAs and shRNA expression constructs may be used to target the entire genomic complement of genes or a subset, such as protein kinases. The format of an individual screen is not only determined by the biological system but also by the choice of RNAi reagent. Both shRNAs and siRNAs are well suited to "one gene per well" screens, in which cells are transfected or infected in multi-well plates (96 or 384), each well containing RNAi reagents that silence one gene (Iorns et al. 2007) (Fig. 1); large scale versions of these screens generally take advantage of automated liquid handling systems. The simplest screens of this type measure cell viability, usually with an ATP colorimetric assay. Potentially, however any biochemical assay adaptable to a high-throughput screen could be used to identify genes involved in the regulation of the particular pathway of interest. More complex and multiple cellular phenotypes can also be assayed using highthroughput microscopy, an approach termed "high-content" analysis. For example, Bakal et al. (2008) recently screened 17,724 RNAi combinations using high-throughput microscopy to measure JNK pathway activity in Drosophila cells. Considerable computational power is required to analyze high-content screens but this approach offers the potential for a much more sophisticated dissection of complex biological networks and pathways.

A

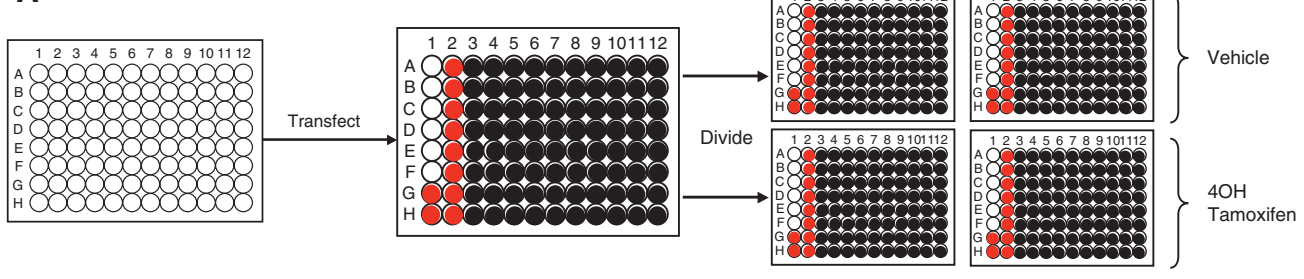

B

C
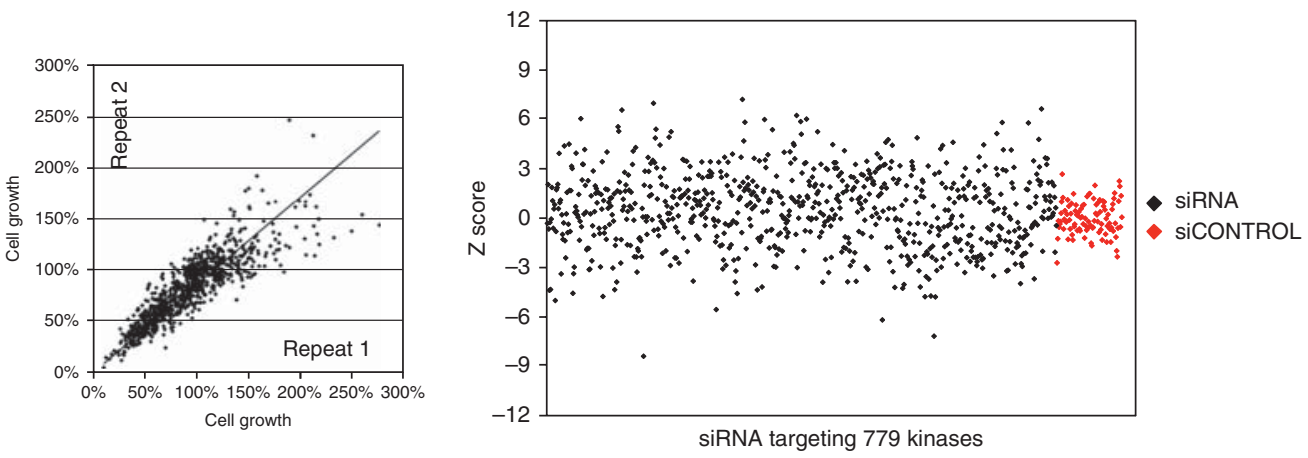

Figure 1. High-throughput siRNA screening (Adapted from Iorns et al. 2008). (A) MCF7 cells plated in 96-well plates were transfected with siRNAs. Each plate contains 80 experimental siRNAs directed against a different gene (black) and 10 nontargeting siControl siRNAs. Transfected cells were divided and half treated with the drug tamoxifen and half with the drug vehicle. After $7 \mathrm{~d}$ cell viability was measured. $(B)$ Reproducibility of the method. Correlation of the effect of each siRNA on viability, in vehicle treated cells, between two duplicate screens. The Spearman correlation coefficient $\mathrm{r}^{2}$ was 0.71. (C) Scatter plot of averaged Z scores from tamoxifen resistance screen carried out in duplicate screens. Black diamonds are experimental siRNAs targeting 779 kinase genes and red diamonds are control siRNAs. 
Single-well siRNA screens of the entire genome $(>20,000$ genes in duplicate or triplicate) are logistically complex and expensive because of the number of assays that need to be performed. An alternative approach is to use shRNA-expression constructs in "pooled" screens (Fig. 2). Pools consisting of up to 5000-10,000 shRNA-expressing vectors are introduced into cells by transfection or infection. Over time some individual shRNAs within the pool, by targeting critical genes, limit or increase growth or viability of the cells into

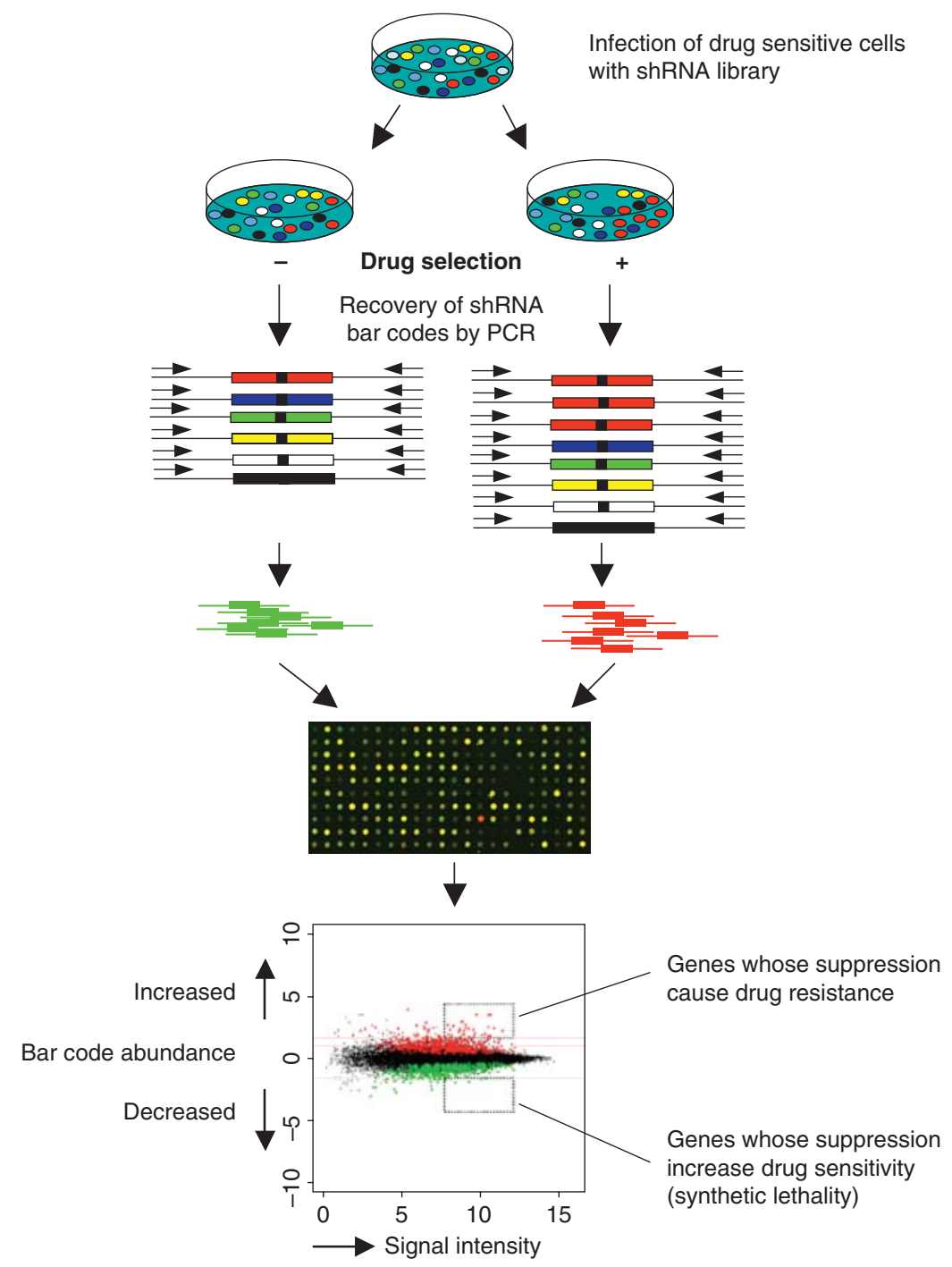

Figure 2. RNAi bar-code screens: Identification of candidate drug response biomarkers. ShRNA bar code loss-of-function genetic screens. Collections of shRNA vectors are expressed polyclonally in drug-sensitive cells and subjected to drug selection. Cells harboring a shRNA vector that confers drug resistance will become enriched in the population, shRNAs that enhance the sensitivity to a cancer drug will become depleted under drug selection compared to a reference population that is not exposed to drug. Each shRNA vector contains a unique identifier sequence (the bar code), which can be recovered by PCR and its abundance quantified on a dedicated DNA micro-array containing the bar code sequences. shRNAs that cause drug resistance are enriched and appear "red" on the micro-array, depleted shRNAs appear "green." 
which they are introduced. A number of methods have been used to identify such shRNAs. The simplest approach, in which the shRNA confers viability on a cell either directly or after challenge with a drug, is to grow out colonies, and rescue the specific shRNA by PCR amplification (Berns et al. 2004). Where direct selection is not appropriate, the entire set of shRNAs can be rescued by PCR amplification of the unique short hairpin sequence of the shRNA vector from the cell population at the start and end of the experiment. Distortions in the frequency of individual hairpins can then be monitored or "deconvoluted" using two methods. In the first deconvolution method, known as barcode screening, the hairpin pool is hybridized to an oligonucleotide microarray to quantify the levels of individual shRNAs (Paddison et al. 2004) (Fig. 2). More recently, next generation sequencing, using platforms such as the Genome Analyzer II (Illumina), has been used for deconvolution as this technology has the ability to simultaneously sequence millions of short pieces of DNA. Both deconvolution strategies can be used to identify shRNA targets that are either selected for (positive selection) or selected against (negative selection).

Selection of both positive and negative controls is critical in defining the magnitude of a meaningful effect and can also inform biologically relevant thresholds for identifying positive hits. Comparison of positive and negative controls also allows the screening window coefficient or $\mathrm{Z}$ factor to be estimated, which describes the suitability of the assay system for large-scale screening. The widely used CellHTS software package developed by Boutros et al. (2006) uses a modification of the $\mathrm{Z}$ score/ median absolute deviation method of defining screen hits. Robust statistical analysis of screens is critical in reducing false positives and negatives and is particularly important if screen data is used for analysis of pathways in packages such as Ingenuity.

Although RNAi screens are now commonplace, there remains significant limitations in their use and interpretation. Most screens so far have been performed in cancer cell lines in culture with all the caveats that this entails and therefore it is critical that any observations be validated in other systems and particularly in clinical samples. In addition, off-target effects causing inhibition of genes, which are not the intended target, are a common problem with RNAi reagents (Echeveri et al. 2006). Therefore "hits" in screens employing pools of siRNAs against a gene target must always be deconvoluted if the results are to be given any credence. Likewise in pooled screens, single shRNA "hits" must be treated with extreme caution. Importantly, these problems may be considerably exacerbated with screens of increasing complexity.

\section{GAIN-OF-FUNCTION GENETIC SCREENS}

When foreign genetic material is used to induce a novel phenotype in a dominant fashion, such genetic screens are referred to as "gain-offunction" screens. In the simplest form, a gain of function screen can consist of the introduction of genomic DNA from one cell into another cell by DNA transfection. More recently, retroviral libraries of cDNAs have been used to induce novel phenotypes. Such libraries have as a major draw back that the abundance of each individual cDNA is a function of the expression in the biological material from which that library was generated. Inevitably, not all genes are expressed in such libraries and these libraries are highly enriched for abundantly expressed genes, limiting their use in functional genetic screens. This problem was avoided elegantly in a recent study by Boehm et al. (2007). They generated a dedicated cDNA collection of 354 activated kinases and used this limited collection of genes to search for genes that could replace phosphatidylinositol 3-kinase (PI3K) signaling in a transformation assay. One of the genes identified was IKBKE. Interestingly, this gene resides in a locus on chromosome 1q32, which is amplified in roughly one third of human primary breast tumors. The contribution of IKBKE to transformation of breast epithelium was underscored by the finding that knockdown of the gene in breast cancer cells having the 1q32 amplicon caused loss of cell viability (Boehm et al. 2007). 
Activation of cellular genes can also be brought about by random insertion of strong enhancers or promoters in the host genome, a technology referred to as insertional mutagenesis. Such insertions are most readily introduced by use of retroviral vectors or by use of transposons. Recently, De et al (2009) used a promoter insertion screen to study mechanisms of resistance to docetaxel in breast cancer. They found that overexpression of the kinesin KIFC3 or the related kinesins KIFC1, KIF1A, or KIF5A in human breast cancer cell lines caused the cells to become more resistant to docetaxel. When all human open reading frames (ORFs) will be available in a sequence-verified and arrayed
Functional Genetics in Breast Cancer Biology

format, gain of function screens will become as mature as loss-of-function screens, and will undoubtedly contribute to the further dissection of the function of hitherto unknown genes in breast cancer biology.

\section{IDENTIFYING NEW GENES IN CANCER PATHWAYS - NEW THERAPEUTIC TARGETS}

RNA interference genetic screens are very suitable to find novel components of cancerrelevant pathways. In the simplest form, such a screen can consist of an artificial luciferase reporter gene, driven by a promoter that responds to pathway activity (Fig. 3). Introduction of the

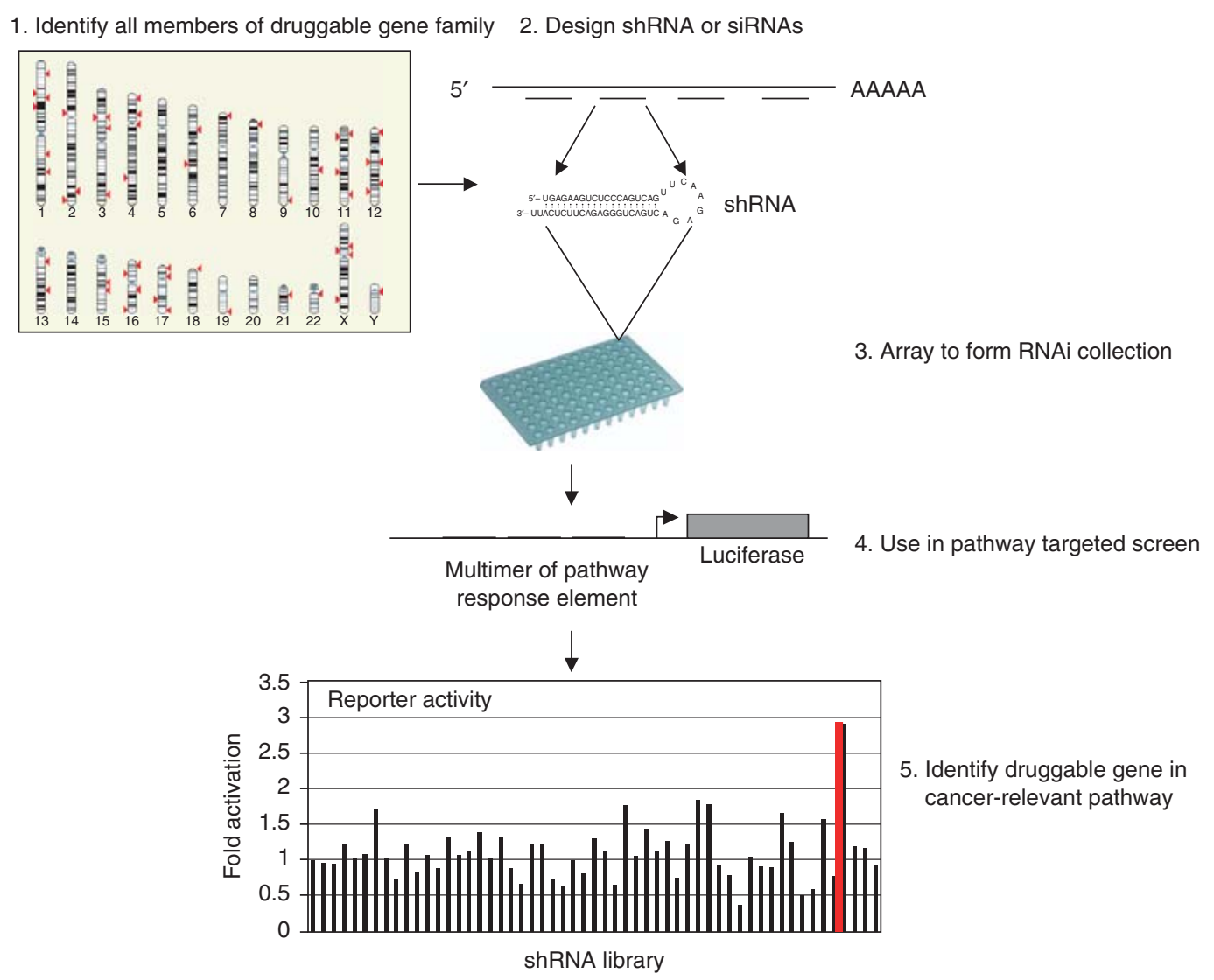

Figure 3. Finding druggable genes in cancer relevant pathways. In a first step, all members of a druggable gene family are identified through bioinformatics approaches. Next, multiple siRNA or shRNA vectors are designed for each of the members of the gene family yielding a gene family knockdown library. This library can then be used to screen for their ability to modulate a cancer-relevant pathway, for instance by using a specific reporter gene construct that responds to pathway activity. 
RNAi reagents in multiwell plates harboring cells expressing the luciferase reporter gene construct can be readily performed in high throughput, allowing one to screen thousands of genes for their potential role in a pathwayof-interest. From a drug development perspective, this type of genetic screen is most productive when performed with selected sets of RNAi reagents that target members of a gene family that are a good starting point for drug discovery. It is well known that some proteins can more be readily inhibited by small molecules than others (e.g., kinases, proteases, and G-protein coupled receptors). Such gene families are considered to be "druggable." By screening families of druggable genes, one can investigate whether any of these preselected genes can modulate a cancer pathway of interest (Fig. 3). If a hit is identified in such a screen, one has an excellent starting point for drug development, as the identified gene is in principle a good starting point for drug development and, by virtue of the way it was discovered, the identified gene is also a critical component of a cancer-relevant signaling pathway. An example if this type of approach is the identification of the cylindromatosis (CYLD) tumor suppressor gene as a regulator of NF- $\mathrm{KB}$ signaling in a RNAi screen targeting a family of proteases. This led to the development of an experimental treatment for this hereditary cancer syndrome using a known inhibitor of NF-кB (Brummelkamp et al. 2003; Oosterkamp et al. 2006).

An alternative way to perform a RNAi screen for novel modulators of a known pathway is the use of a specific small molecule inhibitor of the pathway and screen for genes whose suppression allows cells to overcome pathway inhibition. Examples of such genetic screens are described elsewhere in this article.

\section{GENETICALLY DEFINING CANCER PHENOTYPES}

Novel components of pathways can also be identified by measurement of a downstream phenotypic consequence of signaling through a pathway. Such screens are especially straightforward to carry out when the downstream consequence of the pathway is proliferation arrest or apoptotic cell death. For example, Berns et al. used an engineered primary human cell line harboring a temperature sensitive mutant of the SV40 T antigen to find genes whose suppression causes resistance to a p53-dependent proliferation arrest (Berns et al. 2004). The selection was based on the continued proliferation of cells harboring a specific knockdown vector in the presence of active p53 signaling. In a related screen, secretion of IGFBP7 was found to be essential for induction of senescence by a mutant BRAF oncogene (Wajapeyee et al. 2008). Interestingly, in human metastatic melanoma, IGFBP7 is often epigenetically silenced. Moreover, systemic administration of recombinant IGFBP7 markedly suppressed the growth of metastatic disease and prolonged survival in a mouse model (Wajapeyee et al. 2009). These data highlight that RNAi screens can lead to novel therapeutic interventions that would not have been obvious before the genetic screen was carried out.

An interesting variation on the theme of the simple RNAi screen for genes that act on cell proliferation was performed in colon cancer. In this study, the authors first screened shRNAs targeting about 1000 human genes for their ability to inhibit proliferation of human colon cancer cells. Then, they screened the same set of 1000 genes for their ability to modulate WNT signaling, a pathway that is frequently perturbed in colorectal cancer. Only shRNAs targeting nine genes could both inhibit both proliferation and WNT signaling. This short list of candidate oncogenes was then compared to a list of genes that are located in regions of copy number gain in colon cancer, allowing the identification of cyclin-dependent kinase 8 $(C D K 8)$ as a new colon cancer oncogene (Firestein et al. 2008).

Searching for genes whose suppression confers a fully transformed phenotype onto cells that are only partially transformed has yielded novel tumor suppressor genes. Kolfschoten et al. used large-scale RNAi screens to search for genes that induce anchorage independence in immortalized human fibroblasts (Kolfschoten et al. 2005). They identified PITX1 as a gene 
whose inhibition induces anchorage independent growth through activation of the RAS pathway. Interestingly, low expression of PITX1 in prostate and bladder tumors and in colon cancer cell lines containing wild-type RAS, suggesting that reduced PITX1 expression is an alternative to mutation of RAS in cancer. Along similar lines, a transformation complementation screen of human mammary epithelial cells identified the transcriptional repressor of neuronal gene expression REST as a potential tumor suppressor. Consistent with a tumor suppressor function, REST was found to be a frequent target of deletion in colon cancer (Westbrook et al. 2005).

Induction of apoptosis can be a powerful approach in the treatment of cancer. But which genes are key modulators of apoptosis? Using a set of siRNAs, an inventory was made of the kinases and phosphatases that can modulate apoptosis (Mackeigan et al. 2005). This screen revealed that an unexpectedly large number of phosphatases can regulate apoptosis.

The phenotypes that form the basis for a genetic screen can be more complex than proliferation arrest or apoptosis. As discussed above, sophisticated "high content" screening approaches that take advantage of automated confocal microscopy and image analysis can be used to read complex cellular phenotypes, including subcellular localization of proteins, cell morphology, differentiation and cell migration. Using this approach, novel candidate regulators of mitosis were identified (Moffat et al. 2006).

Arguably the most challenging RNAi screens are those that identify modulators of the metastatic process or genes that control in vivo growth of cells. The notion that only a small subpopulation of cancer stem cells can effectively form a tumor precludes the screening of large collections of vectors in vivo, as the complexity of the library is rapidly lost because of the highly selective outgrowth of a small fraction of the cells. A possible solution to this conundrum is the preselection of a limited number of candidate genes and testing those in vivo. For instance, Gobeil and colleagues used a poorly metastatic mouse melanoma cell to search for genes whose suppression enhanced colony formation in a three-dimensional cell culture system. One of the genes identified, GAS1, was found to be down-regulated in a metastatic subclone of the same melanoma cells. Consistent with a potential metastasis suppressor function, ectopic expression of GAS1 caused suppression of metastasis in a spontaneous metastasis assay (Gobeil et al. 2008). A different approach was used to find new tumor suppressor genes in hepatocellular carcinoma. The authors first searched in human liver cancer samples for regions of copy number loss, arguing that such regions could harbor tumor suppressor genes. This allowed the identification of 362 genes residing in 58 recurrent deletions in liver cancer. ShRNA vectors for these genes were then introduced in partially transformed hepatocytes and small pools of shRNA-transduced cells were injected into mice and scored for tumor growth. This allowed the identification of 6 candidate tumor suppressor genes in liver cancer, only one of which had previously been implicated in human cancer (Zender and Lowe 2008).

\section{STUDYING SENSITIVITY AND RESISTANCE TO CANCER DRUGS}

Many RNAi screens have been carried out with the aim of exploring the mechanism of resistance or sensitivity to drugs used to treat breast and other cancers. These drugs include conventional chemotherapies and anti-endocrine agents as well as more modern molecularly targeted therapeutics. A genome-wide RNAi screen has been performed that identified genes that modify the cellular response to the chemotherapeutic drug paclitaxel (Whitehurst et al. 2007). These genes were divided into functional subgroups including one consisting of proteasome subunits. Interestingly, bortezomib, a proteasome inhibitor, and paclitaxel have previously been shown to be synergistic in patients. This provided proof-of-principle that the screen could identify clinically relevant combination therapies. In addition, a novel potential drug combination was identified in the RNAi screen. A subunit of vacuolar ATPase (V-ATPase) was a 
"hit," which provided a rationale to test the combination of the V-ATPase inhibitor RTA 203 (a salicylihalamide derivative) with paclitaxel; this combination proved to be synergistic. This study highlighted the potential for RNAi screens to identify novel combination therapies. In a similar study, the ceramide-transport protein, COL4A3BP was shown to determine resistance to a number of cytotoxic agents (Swanton et al. 2007). COL4A3BP expression was also suggested to be elevated in drug-resistant cell lines and in paclitaxel-resistant ovarian tumors, suggesting that COL4A3BP expression is a cause of drug resistance and a potential target for use in chemotherapy-resistant cancers (Swanton et al. 2007).

An RNAi screen was used to identify new determinants of response to tamoxifen, a commonly used breast cancer antiendocrine therapy (Iorns et al. 2008) (Fig. 1). MCF7 cells, an estrogen responsive breast cancer cell line, were transfected with siRNAs directed against the kinome and then treated with tamoxifen. Those siRNAs that altered the response to tamoxifen were investigated further. Silencing of the kinase CDK10 was shown to induce resistance to tamoxifen and also other endocrine treatments such as faslodex. Investigation of the mechanism indicated that CDK10 suppression led to up-regulation of the MAP kinase signaling pathway which abrogated the effects of tamoxifen. Importantly, patients with breast cancer treated with tamoxifen whose tumors expressed low levels of CDK10 relapsed significantly earlier than those with high CDK10 expression, suggesting that CDK10 may be a marker of response to tamoxifen. Before this RNAi screen, CDK10 had not been studied in any detail and there was no indication that it might determine response to endocrine therapy. This shows the benefit of RNAi screens in identifying novel components of drug response pathways (Iorns et al. 2007, 2008).

A large-scale RNAi screen was used to discover determinants of trastuzumab (Herceptin) resistance in breast cancer (Berns et al. 2007). This screen identified the phosphatidylinositol 3-kinase (PI3K) pathway as modulating sensitivity to the drug, with mutations in a pathway member, PIK3CA, conferring resistance to trastuzumab. Significantly, this RNAi screen suggests that analysis of the PI3K pathway may provide biomarkers to predict the likelihood of patient response to trastuzumab treatment. A screen for genes influencing sensitivity of cells to the combined HER1/HER2 small molecule inhibitor lapatinib (Tykerb) yielded similar results (Eichhorn et al. 2008).

RNAi screens are also capable of facilitating the development of agents that are in their early stages of development. Brummelkamp and colleagues used a library targeting approximately 8000 genes in a positive-barcode screen to identify mediators of sensitivity to the anticancer drug Nutlin-3, an inhibitor of MDM2 that activates the p53 pathway (Brummelkamp et al. 2006). This resulted in the identification of 53BP1 as a critical mediator of Nutlin-3 induced cytotoxicity. 53BP1 is part of a signaling network induced by DNA damage that is frequently activated in cancer but not in healthy tissues. This led to the proposal that MDM2 inhibitors, such as Nutlin-3, were likely most effective in treating tumors that have both wild-type p53 and activated DNA damage signaling, a substantial fraction of all human tumors. However, the combination of MDM2 inhibitors with DNA-damaging chemotherapies could be harmful to normal cells and may therefore cause undesired side effects (Brummelkamp et al. 2006). In summary, RNAi screens can significantly aid drug development both by identifying mechanisms of sensitivity and resistance and also by indicating potentially potent drug combinations (Iorns et al. 2007).

\section{DISCOVERING POTENTIAL NEW THERAPIES FOR CANCER BY DEFINING SYNTHETIC LETHAL INTERACTIONS}

Synthetic lethality denotes the situation in which two normally nonessential genes become essential when both are lost, or inhibited. Targeting a gene that is synthetically lethal with a cancer-specific mutation should selectively kill tumor cells while sparing normal cells (Kaelin 2005). One of the major advantages of this 
approach is the ability to target cancer cells containing undruggable or loss-of-function mutations in tumor suppressor genes. Conventionally, it has been problematic to devise therapeutic strategies to target cancers with these mutations, as recapitulating tumor suppressor function is technically difficult. Most pharmacological agents inhibit rather than activate protein function and therefore in general cannot be used to target loss-of-function alterations. Identification of synthetic lethal interactions with tumor suppressor genes could allow cells carrying mutations in these genes to be selectively killed. Moreover, the synthetic lethal principle can also be applied to the situation in which tumor cells are strongly addicted to an oncogene driver such as K-RAS (see later).

The use of synthetic lethality to target cancer-specific mutations has been exemplified by the selective killing of cells with $B R C A 1$ or $B R C A 2$ defects using poly(ADP ribose) polymerase (PARP) inhibitors (Ashworth, 2008). These inhibitors showed profound selectivity, being lethal to cells with BRCA1 or BRCA2 deficiency, whereas normal cells are relatively unaffected. Inhibition of PARP leads to the persistence of DNA damage that cannot be repaired in BRCA-deficient cells, which have a specific defect in the homologous recombination DNA repair, but can be processed in normal cells. In this case, the synthetic lethal partners were combined on the basis of suspected mechanisms of action, but most synthetic lethal targets cannot be rationally identified in this manner. However, with the advent of high-throughput RNAi screens it is now possible to perform large-scale synthetic-lethal gene identification in mammalian cells, as is routinely done in model organisms. In yeast, such screens have yielded multiple synthetically lethal interacting genes and genes that are synthetically lethal with small-molecule inhibitors. Although some of these can be extrapolated to mammalian cells, in general pathways and networks in mammalian cells are quite different providing a role for RNAi screening. Using mammalian isogenicpaired cell lines that differ in a single genetic target, RNAi can be used to identify drug targets that when inhibited will result in the selective death of tumor cells.

Three recent synthetic lethal RNAi screens against the same target demonstrate the potential of this approach for the identification of new therapeutic targets directed against cells carrying oncogenic mutations in K-RAS (Barbie et al. 2009; Luo et al. 2009; Scholl et al. 2009). Two distinct approaches were used which exemplify the major technologies for RNAi screens discussed above and different hits were obtained. In the first approach, one group studied the effects of transfecting siRNAs directed against approximately 1000 protein kinase, phosphatase, and cancer-related genes into a panel of eight cell lines, half of which carried oncogenic K-Ras mutations (Scholl et al. 2009). Among a small number of "hits" was gratifyingly, K-RAS itself along with the relatively poorly studied serine-threonine protein kinase STK33. STK33 does not appear to be involved in the RAS signalling network or have any oncogenic activity. Furthermore, STK33 gene does not appear to be frequently mutated or amplified in human cancer, rather K-RAS mutant cells appear to become "rewired" to acquire dependence on STK33. Therefore, it appears that this is indeed a genuine synthetic lethal interaction (Scholl et al. 2009). Another group (Barbie et al. 2009) in a similar approach, used a metanalysis of single well shRNA screens of 19 cell lines to identify TBK1 as a gene required for the viability of K-RAS mutant cells. TBK1 has been implicated in NF-кB signalling suggesting that this pathway is a potential therapeutic target for the treatment of K-RAS driven tumors.

The second approach to identifying targets synthetically lethal with a K-RAS mutation was the use of a genome wide pooled approach targeting about 12,000 genes in a matched pair of isogenic K-RAS mutant and wild-type cells (Luo et al. 2009). Approximately 77 genes revalidated and were confirmed in a secondary screen in a different K-RAS model. Computational methods were used to analyze these genes and assign to cellular pathways. This revealed in increased dependence of K-RAS mutant cells on the mitotic apparatus, including the kinase 
PLK1, and the proteasome. These observations were supported the demonstration that mitotic spindle poisons such as paclitaxel and PLK1 chemical inhibitors were selectively lethal to cells with K-RAS mutations. Potent PLK1 inhibitors are in clinical development and the results of clinical trials of patient populations selected for K-RAS mutation are awaited with interest.

These papers illustrate some of the issues in performing a synthetic lethal screen. On the one hand, isogenic matched paired cell lines are very useful but the downside is that the relevant mutations may not be in the context of other genetic changes, which may permit or co-operate with the change of interest. In contrast, the problem of using unrelated cell lines in which the lesion is either present or absent is that there will be many unrelated genetic changes in each cell, potentially confounding the results. Nevertheless, synthetic lethal RNAi screens are likely to find wide application in the analysis of breast cancer biology and in drug development.

\section{THE FUTURE}

In hardly 10 years, RNA interference evolved from an exotic phenomenon in worms to a major tool for discovery of novel cancer genes. That does not mean that the technology is without flaws. A major issue to date remains the frequency of "off target" effects of siRNAs and shRNAs, caused by inadvertent suppression of other genes than the intended target. It is widely accepted that a "hit" in a screen is a valid "on target" hit if two or more independent RNAi reagents targeting the same transcript cause the same phenotype. However, most siRNA or shRNA collections contain between 2 and 5 RNAi reagents against each gene. Because not all RNAi molecules cause efficient gene suppression, such libraries often still only yield a single RNAi reagent that provokes a phenotype, leaving the issue of "on target" versus "offtarget" unanswered, which often takes considerable time and effort to resolve. Having more complex RNAi libraries with far more RNAi reagents per gene will greatly expedite the identification of on target hits. The recent development of technology for the production of complex nucleic acid libraries using highly parallel in situ oligonucleotide synthesis will likely make such next generation libraries far more efficient and affordable for academic users (Cleary et al. 2004).

The applications for functional genetic screens are likely to considerably expand both in breast cancer biology and drug development (Iorns et al. 2007) but also in investigating the normal mammary gland. How the epithelial cell types in the breast, the luminal and myoepithelial cells, originate from stem cells is still incompletely understood (Smalley and Ashworth 2004) and screens could be used to understand this and the molecular mechanisms specifying lineage commitment. Likewise, in vivo screens could be used to study how the stem cells in the mammary gland, the differentiated cell types and the stromal cells contribute to carcinogenesis. In vivo screens performed in mice should also help identify new cancer genes. Preliminary data suggest that this approach can discover genes affecting mammary cancer cell growth as well as metastasis (A Ashworth, unpublished observations).

As pointed out above, one of the major limitations in drug development is that most genes are not good starting points for drug discovery, leaving only a small fraction of the human genes available as targets of therapeutic intervention. Short duplex RNAs can be used to silence virtually any human gene, vastly increasing the set of targets for cancer therapy. Moreover, using RNAi as a drug allows one to bypass the costly and time-consuming process of small-molecule drug development, a process that fails far more often than it succeeds. If we manage to solve the issues of RNA stability and specific in vivo delivery, RNAi could not only be a tool for drug discovery, but also a powerful therapeutic modality in its own right.

\section{ACKNOWLEDGMENTS}

AA thanks Breakthrough Breast Cancer and Cancer Research UK for research funding and Chris Lord for helpful discussions and assistance with figure preparation. The work of RB 
was supported by grants from the Centre of Biomedical Genetics, the Cancer Genomics Centre, The Netherlands Organisation for Scientific Research (NWO) and the Dutch Cancer Society.

\section{REFERENCES}

Bakal C, Linding R, Llense F, Heffern E, Martin-Blanco E, Pawson T, Perrimon N. 2008. Phosphorylation networks regulating JNK activity in diverse genetic backgrounds. Science 322: 453-456.

Barbie DA, Tamayo P, Boehm JS, Kim SY, Moody SE, Dunn IF, Schinzel AC, Sandy P, Meylan E, Scholl C, et al. 2009. Systematic RNA interference reveals that oncogenic KRAS-driven cancers require TBK1. Nature 462: $108-112$.

Bernards R, Brummelkamp TR, Beijersbergen RL. 2006. shRNA libraries and their use in cancer genetics. Nat Methods 3: 701-706.

Berns K, Hijmans EM, Mullenders J, Brummelkamp TR, Velds A, Heimerikx M, Kerkhoven RM, Madiredjo M, Nijkamp W, Weigelt B, et al. 2004. A large-scale RNAi screen in human cells identifies new components of the p53 pathway. Nature 428: 431-437.

Berns K, Horlings HM, Hennessy BT, Madiredjo M, Hijmans EM, Beelen K, Linn SC, Gonzalez-Angulo AM, Stemke-Hale K, Hauptmann M, et al. 2007. A functional genetic approach identifies the PI3K pathway as a major determinant of trastuzumab resistance in breast cancer. Cancer Cell 12: 395-402.

Boehm JS, Zhao JJ, Yao J, Kim SY, Firestein R, Dunn IF, Sjostrom SK, Garraway LA, Weremowicz S, Richardson AL, et al. 2007. Integrative genomic approaches identify IKBKE as a breast cancer oncogene. Cell 129: 1065-1079.

Boutros M, Bras LP, Huber W. 2006. Analysis of cell-based RNAi screens. Genome Biol 7: R66.

Brummelkamp TR, Nijman SM, Dirac AM, Bernards R. 2003. Loss of the cylindromatosis tumour suppressor inhibits apoptosis by activating NF-kappaB. Nature 424: 797-801.

Brummelkamp TR, Fabius AW, Mullenders J, Madiredjo M, Velds A, Kerkhoven RM, Bernards R, Beijersbergen RL. 2006. An shRNA barcode screen provides insight into cancer cell vulnerability to MDM2 inhibitors. Nat Chem Biol 2: 202-206.

Cleary MA, Kilian K, Wang Y, Bradshaw J, Cavet G, Ge W, Kulkarni A, Paddison PJ, Chang K, Sheth N, et al. 2004. Production of complex nucleic acid libraries using highly parallel in situ oligonucleotide synthesis. Nat Methods 1: 241-248.

De S, Cipriano R, Jackson MW, Stark GR. 2009. Overexpression of kinesins mediates docetaxel resistance in breast cancer cells. Cancer Res 69: 8035-8042.

Echeverri CJ, Beachy PA, Baum B, Boutros M, Buchholz F, Chanda SK, Downward J, Ellenberg J, Fraser AG, Hacohen N, et al. 2006. Minimizing the risk of reporting false positives in large-scale RNAi screens. Nat Methods 3: 777-779.
Eichhorn PJ, Gili M, Scaltriti M, Serra V, Guzman M, Nijkamp W, Beijersbergen RL, Valero V, Seoane J, Bernards $\mathrm{R}$, et al. 2008. Phosphatidylinositol 3-kinase hyperactivation results in lapatinib resistance that is reversed by the mTOR/phosphatidylinositol 3-kinase inhibitor NVPBEZ235. Cancer Res 68: 9221-9230.

Firestein R, Bass AJ, Kim SY, Dunn IF, Silver SJ, Guney I, Freed E, Ligon AH, Vena N, Ogino S, et al. 2008. CDK8 is a colorectal cancer oncogene that regulates beta-catenin activity. Nature 455: 547-551.

Gobeil S, Zhu X, Doillon CJ, Green MR. 2008. A genomewide shRNA screen identifies GAS1 as a novel melanoma metastasis suppressor gene. Genes Dev 22: 2932-2940.

Iorns E, Lord CJ, Turner N, Ashworth A. 2007. Utilizing RNA interference to enhance cancer drug discovery. Nat Rev Drug Discov 6: 556-568.

Iorns E, Turner NC, Elliott R, Syed N, Garrone O, Gasco M, Tutt AN, Crook T, Lord CJ, Ashworth A. 2008. Identification of CDK10 as an important determinant of resistance to endocrine therapy for breast cancer. Cancer Cell 13: 91-104.

Kaelin WG, Jr. 2005. The concept of synthetic lethality in the context of anticancer therapy. Nat Rev Cancer 5: 689-698.

Kittler R, Surendranath V, Heninger AK, Slabicki M, Theis M, Putz G, Franke K, Caldarelli A, Grabner H, Kozak $\mathrm{K}$, et al. 2007. Genome-wide resources of endoribonuclease-prepared short interfering RNAs for specific loss-of-function studies. Nat Methods 4: 337-344.

Kolfschoten IG, van Leeuwen B, Berns K, Mullenders J, Beijersbergen RL, Bernards R, Voorhoeve PM, Agami R. 2005. A genetic screen identifies PITX1 as a suppressor of RAS activity and tumorigenicity. Cell 121: 849-858.

Luo J, Emanuele ML, Li D, Creighton CJ, Schlabach MR, Westbrook TF, Wong KK, Elledge SJ. 2009. A genomewide RNAi screen identifies multiple synthetic lethal interactions with the Ras oncogene. Cell 137: 835-848.

Mackeigan JP, Murphy LO, Blenis J. 2005. Sensitized RNAi screen of human kinases and phosphatases identifies new regulators of apoptosis and chemoresistance. Nat Cell Biol 7: 591-600.

Moffat J, Grueneberg DA, Yang X, Kim SY, Kloepfer AM, Hinkle G, Piqani B, Eisenhaure TM, Luo B, Grenier JK, et al. 2006. A Lentiviral RNAi Library for Human and Mouse Genes Applied to an Arrayed Viral High-Content Screen. Cell 124: 1283-1298.

Oosterkamp HM, Neering H, Nijman SM, Dirac AM, Mooi WJ, Bernards R, Brummelkamp TR. 2006. An evaluation of the efficacy of topical application of salicylic acid for the treatment of familial cylindromatosis. Br J Dermatol 155: $182-185$.

Paddison PJ, Silva JM, Conklin DS, Schlabach M, Li M, Aruleba S, Balija V, O'Shaughnessy A, Gnoj L, Scobie K, et al. 2004. A resource for large-scale RNA-interferencebased screens in mammals. Nature 428: 427-431.

Schlabach MR, Luo J, Solimini NL, Hu G, Xu Q, Li MZ, Zhao Z, Smogorzewska A, Sowa ME, Ang XL, et al. 2008. Cancer proliferation gene discovery through functional genomics. Science 319: 620-624.

Scholl C, Frohling S, Dunn IF, Schinzel AC, Barbie DA, Kim SY, Silver SJ, Tamayo P, Wadlow RC, Ramaswamy S, et al. 2009. Synthetic lethal interaction between oncogenic 
A. Ashworth and R. Bernards

KRAS dependency and STK33 suppression in human cancer cells. Cell 137: 821-834.

Smalley M, Ashworth A. 2004 Stem cells and breast cancer: A field in transit. Nat Rev Cancer 3: 832-844.

Swanton C, Marani M, Pardo O, Warne PH, Kelly G, Sahai E, Elustondo F, Chang J, Temple J, Ahmed AA, et al. 2007. Regulators of mitotic arrest and ceramide metabolism are determinants of sensitivity to paclitaxel and other chemotherapeutic drugs. Cancer Cell 11: 498-512.

van 't Veer LJ, Dai H, van de Vijver MJ, He YD, Hart AA, Mao M, Peterse HL, van der Kooy K, Marton MJ, Witteveen AT, et al. 2002. Gene expression profiling predicts clinical outcome of breast cancer. Nature 415: 530-536.

Wajapeyee N, Kapoor V, Mahalingam M, Green MR. 2009. Efficacy of IGFBP7 for treatment of metastatic melanoma and other cancers in mouse models and human cell lines. Mol Cancer Therapeutics 8: 3009-3014.
Wajapeyee N, Serra RW, Zhu X, Mahalingam M, Green MR 2008. Oncogenic BRAF induces senescence and apoptosis through pathways mediated by the secreted protein IGFBP7. Cell 132: 363-374.

Westbrook TF, Martin ES, Schlabach MR, Leng Y, Liang AC, Feng B, Zhao JJ, Roberts TM, Mandel G, Hannon GJ, et al. 2005. A genetic screen for candidate tumor suppressors identifies REST. Cell 121: 837-848.

Whitehurst AW, Bodemann BO, Cardenas J, Ferguson D, Girard L, Peyton M, Minna JD, Michnoff C, Hao W, Roth MG, et al. 2007. Synthetic lethal screen identification of chemosensitizer loci in cancer cells. Nature 446: 815-819.

Zender L, Lowe SW. 2008. Integrative oncogenomic approaches for accelerated cancer-gene discovery. Curr opin oncol 20: 72-76. 


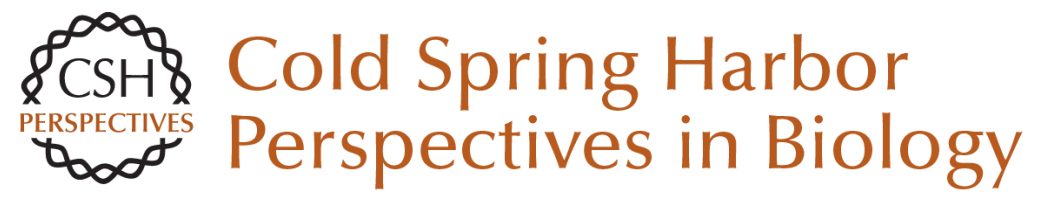

\title{
Using Functional Genetics to Understand Breast Cancer Biology
}

\author{
Alan Ashworth and Rene Bernards
}

Cold Spring Harb Perspect Biol 2010; doi: 10.1101/cshperspect.a003327 originally published online June 2, 2010

\section{Subject Collection The Mammary Gland as an Experimental Model}

On the Role of the Microenvironment in Mammary Gland Development and Cancer Derek Radisky

On Using Functional Genetics to Understand Breast Cancer Biology Kornelia Polyak

On Oncogenes and Tumor Suppressor Genes in the Mammary Gland Rushika M. Perera and Nabeel Bardeesy

On Leukocytes in Mammary Development and Cancer Cyrus M. Ghajar

On Chromatin Remodeling in Mammary Gland Differentiation and Breast Tumorigenesis Kornelia Polyak

On Hormone Action in the Mammary Gland J.M. Rosen

TGF- $\beta$ Biology in Mammary Development and Breast Cancer

Harold Moses and Mary Helen Barcellos-Hoff

A Compendium of the Mouse Mammary Tumor Biologist: From the Initial Observations in the House Mouse to the Development of Genetically Engineered Mice Robert D. Cardiff and Nicholas Kenney
On How Mammary Gland Reprogramming Metalloproteinases Couple Form with Function Bonnie F. Sloane

On Molecular Mechanisms Guiding Embryonic Mammary Gland Development Gertraud W. Robinson

On Stem Cells in the Human Breast Mark A. LaBarge

On Murine Mammary Epithelial Stem Cells:

Discovery, Function, and Current Status Jeffrey M. Rosen

On In Vivo Imaging in Cancer David Piwnica-Worms

Choosing a Mouse Model: Experimental Biology in Context--The Utility and Limitations of Mouse Models of Breast Cancer Alexander D. Borowsky

Mammary Gland ECM Remodeling, Stiffness, and Mechanosignaling in Normal Development and Tumor Progression Pepper Schedin and Patricia J. Keely

Molecular Mechanisms Guiding Embryonic Mammary Gland Development Pamela Cowin and John Wysolmerski

For additional articles in this collection, see http://cshperspectives.cshlp.org/cgi/collection/

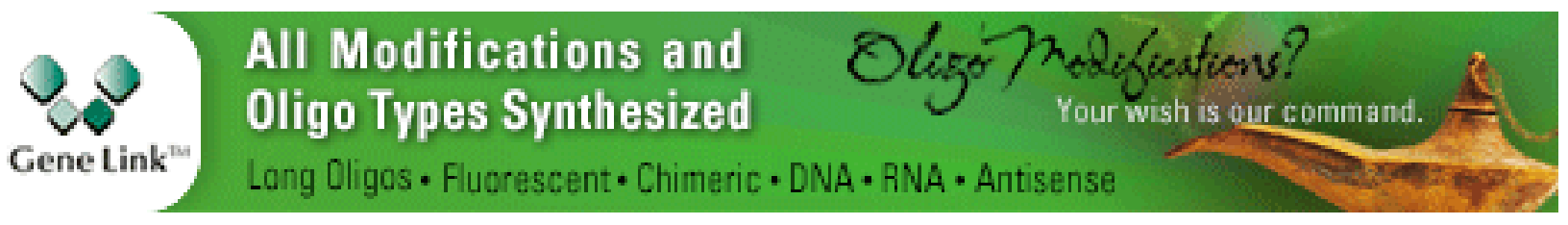


For additional articles in this collection, see http://cshperspectives.cshlp.org/cgi/collection/

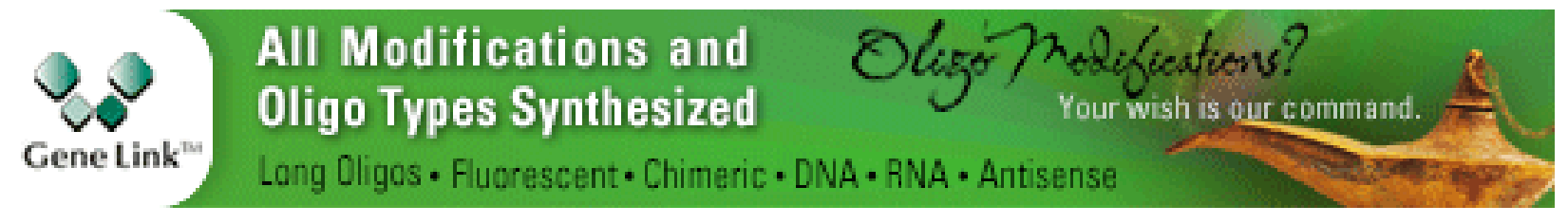

Copyright @ 2010 Cold Spring Harbor Laboratory Press; all rights reserved 